\title{
Optimal inequalities for the Casorati curvatures of submanifolds of real space forms endowed with semi-symmetric metric connections
}

\author{
Chul Woo Lee ${ }^{1}$, Dae Won Yoon ${ }^{2}$ and Jae Won Lee $3^{3^{*}}$
}

"Correspondence:

jaewon07@gmail.com ${ }^{3}$ Department of Mathematics

Education, Busan National Univeristy of Education, Busan, 611-736, South Korea

Full list of author information is available at the end of the article

\begin{abstract}
In this paper, we prove two optimal inequalities involving the intrinsic scalar curvature and extrinsic Casorati curvature of submanifolds of real space forms endowed with a semi-symmetric metric connection. Moreover, we show that in both cases, the equality at all points characterizes the invariantly quasi-umbilical submanifolds. MSC: 53C40; 53B05
\end{abstract}

Keywords: Casorati curvature; real space form; semi-symmetric metric connection

\section{Introduction}

The idea of a semi-symmetric linear connection on a differentiable manifold was introduced by Friedmann and Schouten in [1]. The notion of a semi-symmetric metric connection on a Riemannian manifold was introduced by Hayden in [2]. Later, Yano in [3] studied some properties of a Riemannian manifold endowed with a semi-symmetric metric connection. In [4, 5], Imai found some properties of a Riemannian manifold and a hypersurface of a Riemannian manifold with a semi-symmetric metric connection. Nakao in [6] studied submanifolds of a Riemannian manifold with semi-symmetric metric connections.

On the other hand, the theory of Chen invariants, initiated by Chen [7] in a seminal paper published in 1993, is presently one of the most interesting research topic in differential geometry of submanifolds. Chen established a sharp inequality for a submanifold in a real space form using the scalar curvature and the sectional curvature, and the squared mean curvature. That is, he established simple relationships between the main intrinsic invariants and the main extrinsic invariants of a submanifold in real space forms with any codimensions in [8]. Many famous results concerned Chen invariants and inequalities for the different classes of submanifolds in various ambient spaces, like complex space forms [9-11]. Recently, in [12,13], Mihai and Özgür proved Chen inequalities for submanifolds of real, complex, and Sasakian space forms endowed with semi-symmetric metric connections and in [14, 15], Özgür and Murathan gave Chen inequalities for submanifolds of a locally conformal almost cosymplectic manifold and a cosymplectic space form endowed with semi-symmetric metric connections. Moreover, Zhang et al. [16] obtained Chen-like 
inequalities for submanifolds of a Riemannian manifold of quasi-constant curvature endowed with a semi-symmetric metric connection by using an algebraic approach.

Instead of concentrating on the sectional curvature with the extrinsic squared mean curvature, the Casorati curvature of a submanifold in a Riemannian manifold was considered as an extrinsic invariant defined as the normalized square of the length of the second fundamental form. The notion of Casorati curvature extends the concept of the principal direction of a hypersurface of a Riemannian manifold. Several geometers in [17-21] found geometrical meaning and the importance of the Casorati curvature. Therefore, it is of great interest to obtain optimal inequalities for the Casorati curvatures of submanifolds in different ambient spaces. Decu et al. in [22] obtained some optimal inequalities involving the scalar curvature and the Casorati curvature of a Riemannian submanifold in a real space form and the holomorphic sectional curvature and the Casorati curvature of a Kähler hypersurface in a complex space form. They also proved an inequality in which the scalar curvature is estimated from above by the normalized Casorati curvatures in [23]. Recently, some optimal inequalities involving Casorati curvatures were proved in [24, 25] for slant submanifolds in quaternionic space forms.

As a natural prolongation of our research, in this paper we will study these inequalities for submanifolds in real space forms, endowed with semi-symmetric metric connections.

Theorem 1.1 Let $M^{n}$ be a submanifold of a real space form $N^{m}(c)$ with a semi-symmetric metric connection. Then:

(i) The normalized $\delta$-Casorati curvature $\delta_{C}(n-1)$ satisfies

$$
\rho \leq \delta_{C}(n-1)+c-\frac{2}{n} \operatorname{trace}(\alpha) .
$$

Moreover, the equality sign holds if and only if $M^{n}$ is an invariantly quasi-umbilical submanifold with trivial normal connection in $N^{m}(c)$, such that with respect to suitable orthonormal tangent frame $\left\{\xi_{1}, \ldots, \xi_{n}\right\}$ and normal orthonormal frame $\left\{\xi_{n+1}, \ldots, \xi_{m}\right\}$, the shape operators $A_{r} \equiv A_{\xi_{r}}, r \in\{n+1, \ldots, m\}$, take the following forms:

$$
A_{n+1}=\left(\begin{array}{cccccc}
a & 0 & 0 & \cdots & 0 & 0 \\
0 & a & 0 & \cdots & 0 & 0 \\
0 & 0 & a & \cdots & 0 & 0 \\
\vdots & \vdots & \vdots & \ddots & \vdots & \vdots \\
0 & 0 & 0 & \cdots & a & 0 \\
0 & 0 & 0 & \cdots & 0 & 2 a
\end{array}\right), \quad A_{n+2}=\cdots=A_{m}=0
$$

(ii) The normalized $\delta$-Casorati curvature $\hat{\delta}_{C}(n-1)$ satisfies

$$
\rho \leq \hat{\delta}_{C}(n-1)+c-\frac{2}{n} \operatorname{trace}(\alpha) .
$$

Moreover, the equality sign holds if and only if $M^{n}$ is an invariantly quasi-umbilical submanifold with trivial normal connection in $N^{m}(c)$, such that with respect to suitable orthonormal tangent frame $\left\{\xi_{1}, \ldots, \xi_{n}\right\}$ and normal orthonormal frame 
$\left\{\xi_{n+1}, \ldots, \xi_{m}\right\}$, the shape operators $A_{r} \equiv A_{\xi_{r}}, r \in\{n+1, \ldots, m\}$, take the following forms:

$$
A_{n+1}=\left(\begin{array}{cccccc}
2 a & 0 & 0 & \cdots & 0 & 0 \\
0 & 2 a & 0 & \cdots & 0 & 0 \\
0 & 0 & 2 a & \cdots & 0 & 0 \\
\vdots & \vdots & \vdots & \ddots & \vdots & \vdots \\
0 & 0 & 0 & \cdots & 2 a & 0 \\
0 & 0 & 0 & \cdots & 0 & a
\end{array}\right), \quad A_{n+2}=\cdots=A_{m}=0
$$

\section{Preliminaries}

Let $N^{m}$ be an $m$-dimensional Riemannian manifold and $\widetilde{\nabla}$ a linear connection on $N^{m}$. If the torsion tensor $\widetilde{T}$ of $\widetilde{\nabla}$, defined by

$$
\widetilde{T}(\widetilde{X}, \widetilde{Y})=\widetilde{\nabla}_{\widetilde{X}} \widetilde{Y}-\widetilde{\nabla}_{\widetilde{Y}} \widetilde{X}-[\widetilde{X}, \widetilde{Y}]
$$

for any vector field $\widetilde{X}$ and $\tilde{Y}$ on $N^{m}$, satisfies

$$
\widetilde{T}(\widetilde{X}, \tilde{Y})=\phi(\widetilde{Y}) \widetilde{X}-\phi(\widetilde{X}) \widetilde{Y}
$$

for a 1 -form $\phi$, then the connection $\widetilde{\nabla}$ is called a semi-symmetric connection.

Let $g$ be a Riemannian metric on $N^{m}$. If $\widetilde{\nabla} g=0$, then $\widetilde{\nabla}$ is called a semi-symmetric metric connection on $N^{m}$.

Following [3], a semi-symmetric metric connection $\widetilde{\nabla}$ on $N^{m}$ is given by

$$
\widetilde{\nabla} \widetilde{X}=\tilde{\nabla}_{\widetilde{X}} \widetilde{Y}+\phi(\widetilde{Y}) \widetilde{X}-g(\widetilde{X}, \widetilde{Y}) P
$$

for any vector fields $\widetilde{X}$ and $\tilde{Y}$ on $N^{m}$, where $\stackrel{\mathscr{\nabla}}{\nabla}$ denotes the Levi-Civita connection with respect to the Riemannian metric $g$ and $P$ is a vector field defined by $g(P, \widetilde{X})=\phi(\tilde{X})$, for any vector field $\tilde{X}$.

We will consider a Riemannian manifold $N^{m}$ endowed with a semi-symmetric metric connection $\widetilde{\nabla}$ and the Levi-Civita connection denoted by $\stackrel{2}{\nabla}$.

Let $M^{n}$ be an $n$-dimensional submanifold of an $m$-dimensional Riemannian manifold $N^{m}$. On the submanifold $M^{n}$, we consider the induced semi-symmetric metric connection, denoted by $\nabla$ and the induced Levi-Civita connection, denoted by $\stackrel{\circ}{\nabla}$.

Let $\widetilde{R}$ be the curvature tensor of $N^{m}$ with respect to $\widetilde{\nabla}$ and $\widetilde{R}$ the curvature tensor of $N^{m}$ with respect to $\stackrel{e}{\nabla}$. We also denote by $R$ and $\stackrel{R}{R}$ the curvature tensors of $\nabla$ and $\stackrel{\circ}{\nabla}$, respectively, on $M^{n}$.

The Gauss formulas with respect to $\nabla$ and $\nabla^{\circ}$, respectively, can be written as

$$
\begin{array}{ll}
\widetilde{\nabla}_{X} Y=\nabla_{X} Y+h(X, Y), & X, Y \in \chi\left(M^{n}\right), \\
\stackrel{\circ}{\nabla}_{X} Y=\stackrel{\circ}{\nabla}_{X} Y+\stackrel{\circ}{h}(X, Y), & X, Y \in \chi\left(M^{n}\right),
\end{array}
$$

where $\stackrel{\circ}{h}$ is the second fundamental form of $M^{n}$ in $N^{m}$ and $h$ is a $(0,2)$-tensor on $M^{n}$. According to the formula (7) from [6], $h$ is also symmetric. One denotes by $\stackrel{H}{H}$ the mean 
curvature vector of $M^{n}$ and $N^{m}$. Let $N^{m}(c)$ be a real space form of constant sectional curvature $c$ endowed with a semi-symmetric metric connection $\widetilde{\nabla}$.

The curvature tensor $\stackrel{\mathscr{R}}{R}$ with respect to the Levi-Civita connection $\widetilde{\nabla}$ on $N^{m}(c)$ is expressed by

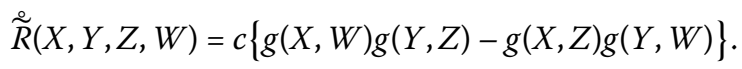

Then the curvature tensor $\widetilde{R}$ with respect to the semi-symmetric metric connection $\widetilde{\nabla}$ on $N^{m}(c)$ can be written as [5]

$$
\begin{aligned}
\widetilde{R}(X, Y, Z, W)= & \stackrel{\mathscr{R}}{R}(X, Y, Z, W)-\alpha(Y, Z) g(X, W) \\
& +\alpha(X, Z) g(Y, W)-\alpha(X, W) g(Y, Z) \\
& +\alpha(Y, W) g(X, Z)
\end{aligned}
$$

for any vector fields $X, Y, Z, W \in \chi\left(M^{n}\right)$, where $\alpha$ is a $(0,2)$-tensor field defined by

$$
\alpha(X, Y)=\left(\stackrel{\sim}{\nabla}_{X} \phi\right) Y-\phi(X) \phi(Y)+\frac{1}{2} \phi(P) g(X, Y), \quad \forall X, Y \in \chi\left(M^{n}\right) .
$$

From (2.1) and (2.2), it follows that the curvature tensor $\widetilde{R}$ can be expressed as

$$
\begin{aligned}
\widetilde{R}(X, Y, Z, W)= & c\{g(X, W) g(Y, Z)-g(X, Z) g(Y, W)\} \\
& -\alpha(Y, Z) g(X, W)+\alpha(X, Z) g(Y, W) \\
& -\alpha(X, W) g(Y, Z)+\alpha(Y, W) g(X, Z) .
\end{aligned}
$$

Denote by $\lambda$ the trace of $\alpha$.

The Gauss equation for the submanifold $M^{n}$ in the real space form $N^{m}(c)$ is

$$
\begin{aligned}
& \stackrel{乛}{R}(X, Y, Z, W)=\stackrel{\circ}{R}(X, Y, Z, W)+g(\stackrel{\circ}{h}(X, Z), \stackrel{h}{h}(Y, W)) \\
& -g(\stackrel{h}{h}(X, W), \stackrel{\circ}{h}(Y, Z)) \text {. }
\end{aligned}
$$

Let $\pi \subset T_{x} M^{n}, x \in M^{n}$, be a 2-plane section. Denote by $K(\pi)$ the sectional curvature of $M^{n}$ with respect to the induced semi-symmetric metric connection $\nabla$. For any orthonormal basis $\left\{e_{1}, \ldots, e_{n}\right\}$ of the tangent space $T_{x} M^{n}$ and $\left\{e_{n+1}, \ldots, e_{m}\right\}$ is an orthonormal basis of the normal space $T_{x}^{\perp} M$, then the scalar curvature $\tau$ at $x$ is defined by

$$
\tau(x)=\sum_{1 \leq i<j \leq n} K\left(e_{i} \wedge e_{j}\right)
$$

and the normalized scalar curvature $\rho$ of $M$ is defined by

$$
\rho=\frac{2 \tau}{n(n-1)}
$$

We denote by $H$ the mean curvature vector, that is,

$$
H(x)=\frac{1}{n} \sum_{i=1}^{n} h\left(e_{i}, e_{i}\right),
$$


and we also set

$$
h_{i j}^{\alpha}=g\left(h\left(e_{i}, e_{j}\right), e_{\alpha}\right), \quad i, j \in\{1, \ldots, n\}, \alpha \in\{n+1, \ldots, m\} .
$$

Then the squared mean curvature of the submanifold $M$ in $N$ is defined by

$$
\|H\|^{2}=\frac{1}{n^{2}} \sum_{\alpha=n+1}^{m}\left(\sum_{i=1}^{n} h_{i i}^{\alpha}\right)^{2},
$$

and the squared norm of $h$ over dimension $n$ is denoted by $\mathcal{C}$ and is called the Casorati curvature of the submanifold $M$. Therefore, we have

$$
\mathcal{C}=\frac{1}{n} \sum_{\alpha=n+1}^{m} \sum_{i, j=1}^{n}\left(h_{i j}^{\alpha}\right)^{2} .
$$

The submanifold $M$ is called invariantly quasi-umbilical if there exist $m-n$ mutually orthogonal unit normal vectors $\xi_{n+1}, \ldots, \xi_{m}$ such that the shape operators with respect to all directions $\xi_{\alpha}$ have an eigenvalue of multiplicity $n-1$ and that for each $\xi_{\alpha}$ the distinguished eigendirection is the same [26].

Suppose now that $L$ is an $r$-dimensional subspace of $T_{x} M, r \geq 2$, and $\left\{e_{1}, \ldots, e_{r}\right\}$ be an orthonormal basis of $L$. Then the scalar curvature $\tau(L)$ of the $r$-plane section $L$ is given by

$$
\tau(L)=\sum_{1 \leq \alpha<\beta \leq r} K\left(e_{\alpha} \wedge e_{\beta}\right)
$$

and the Casorati curvature $\mathcal{C}(L)$ of the subspace $L$ is defined as

$$
\mathcal{C}(L)=\frac{1}{r} \sum_{\alpha=n+1}^{m} \sum_{i, j=1}^{r}\left(h_{i j}^{\alpha}\right)^{2} .
$$

The normalized $\delta$-Casorati curvature $\delta_{c}(n-1)$ and $\hat{\delta}_{c}(n-1)$ are given by

$$
\left[\delta_{c}(n-1)\right]_{x}=\frac{1}{2} \mathcal{C}_{x}+\frac{n+1}{2 n} \inf \left\{\mathcal{C}(L) \mid L: \text { a hyperplane of } T_{x} M\right\}
$$

and

$$
\left[\hat{\delta}_{c}(n-1)\right]_{x}=2 \mathcal{C}_{x}-\frac{2 n-1}{2 n} \sup \left\{\mathcal{C}(L) \mid L: \text { a hyperplane of } T_{x} M\right\} .
$$

\section{Proof of the theorem}

From [6], the Gauss equation with respect to the semi-symmetric metric connection is

$$
\begin{aligned}
\widetilde{R}(X, Y, Z, W)= & R(X, Y, Z, W)+g(h(X, Z), h(Y, W)) \\
& -g(h(Y, Z), h(X, W)) .
\end{aligned}
$$

Let $x \in M^{n}$ and $\left\{e_{1}, e_{2}, \ldots, e_{n}\right\}$ and $\left\{e_{n+1}, \ldots, e_{m}\right\}$ be orthonormal basis of $T_{x} M^{n}$ and $T_{x}^{\perp} M^{n}$, respectively. For $X=W=e_{i}, Y=Z=e_{j}, i \neq j$, from (2.3), it follows that

$$
\widetilde{R}\left(e_{i}, e_{j}, e_{j}, e_{i}\right)=c-\alpha\left(e_{i}, e_{i}\right)-\alpha\left(e_{j}, e_{j}\right) .
$$


From (3.1) and (3.2), we get

$$
\begin{aligned}
c- & \alpha\left(e_{i}, e_{i}\right)-\alpha\left(e_{j}, e_{j}\right) \\
& =R\left(e_{i}, e_{j}, e_{j}, e_{i}\right)+g\left(h\left(e_{i}, e_{j}\right), h\left(e_{i}, e_{j}\right)\right)-g\left(h\left(e_{i}, e_{i}\right), h\left(e_{j}, e_{j}\right)\right) .
\end{aligned}
$$

By summation over $1 \leq i, j \leq n$, it follows from the previous relation that

$$
2 \tau=n^{2}\|H\|^{2}-n \mathcal{C}+n(n-1) c-2(n-1) \operatorname{trace}(\alpha) .
$$

We define now the following function, denoted by $\mathcal{P}$, which is a quadratic polynomial in the components of the second fundamental form:

$$
\mathcal{P}=\frac{1}{2} n(n-1) \mathcal{C}+\frac{1}{2}(n+1)(n-1) \mathcal{C}(L)-2 \tau+n(n-1) c-2(n-1) \operatorname{trace}(\alpha)
$$

Without loss of generality, by assuming that $L$ is spanned by $e_{1}, \ldots, e_{n-1}$, one derives that

$$
\begin{aligned}
\mathcal{P}= & \frac{n+1}{2} \sum_{\alpha=n+1}^{m}\left(\sum_{i, j=1}^{n}\left(h_{i j}^{\alpha}\right)^{2}\right)+\frac{n+1}{2} \sum_{\alpha=n+1}^{m}\left(\sum_{i, j=1}^{n-1}\left(h_{i j}^{\alpha}\right)^{2}\right) \\
& -\sum_{\alpha=n+1}^{m}\left(\sum_{i=1}^{n} h_{i i}^{\alpha}\right)^{2}
\end{aligned}
$$

and now we easily obtain

$$
\begin{aligned}
\mathcal{P}= & \sum_{\alpha=n+1}^{m} \sum_{i=1}^{n-1}\left[n\left(h_{i i}^{\alpha}\right)^{2}+(n+1)\left(h_{i n}^{\alpha}\right)^{2}\right] \\
& +\sum_{\alpha=n+1}^{m}\left[2(n+1) \sum_{i<j=1}^{n-1}\left(h_{i j}^{\alpha}\right)^{2}-2 \sum_{i<j=1}^{n} h_{i i}^{\alpha} h_{j j}^{\alpha}+\frac{n-1}{2}\left(h_{n n}^{\alpha}\right)^{2}\right] .
\end{aligned}
$$

From (3.4), it follows that the critical points

$$
h^{c}=\left(h_{11}^{n+1}, h_{12}^{n+1}, \ldots, h_{n n}^{n+1}, \ldots, h_{11}^{m}, \ldots, h_{n n}^{m}\right)
$$

of $\mathcal{P}$ are the solutions of the following system of linear homogeneous equations:

$$
\left\{\begin{array}{l}
\frac{\partial \mathcal{P}}{\partial h_{i i}^{\alpha}}=2(n+1) h_{i i}^{\alpha}-2 \sum_{k=1}^{n} h_{k k}^{\alpha}=0, \\
\frac{\partial \mathcal{P}}{\partial h_{n n}^{\alpha}}=(n-1) h_{n n}^{\alpha}-2 \sum_{k=1}^{n-1} h_{k k}^{\alpha}=0, \\
\frac{\partial \mathcal{P}}{\partial h_{i j}^{\alpha}}=4(n+1) h_{i j}^{\alpha}=0, \\
\frac{\partial \mathcal{P}}{\partial h_{i n}^{\alpha}}=2(n+1) h_{i n}^{\alpha}=0,
\end{array}\right.
$$

with $i, j \in\{1, \ldots, n-1\}, i \neq j$ and $\alpha \in\{n+1, \ldots, m\}$. Thus, every solution $h^{c}$ has $h_{i j}^{\alpha}=0$ for $i \neq j$, and the determinant which corresponds to the first two sets of equations of the above system is zero (there exist solutions for non-totally geodesic submanifolds). Moreover, it 
is easy to see that the Hessian matrix of $\mathcal{P}$ has the form

$$
\mathcal{H}(\mathcal{P})=\left(\begin{array}{ccc}
H_{1} & \mathbf{0} & \mathbf{0} \\
\mathbf{0} & H_{2} & \mathbf{0} \\
\mathbf{0} & \mathbf{0} & H_{3}
\end{array}\right),
$$

where

$$
H_{1}=\left(\begin{array}{ccccc}
2 n & -2 & \cdots & -2 & -2 \\
-2 & 2 n & \cdots & -2 & -2 \\
\vdots & \vdots & \ddots & \vdots & \vdots \\
-2 & -2 & \cdots & 2 n & -2 \\
-2 & -2 & \cdots & -2 & n-1
\end{array}\right),
$$

$\mathbf{0}$ denotes the null matrix of corresponding dimensions and $H_{2}, H_{3}$ are the next diagonal matrices

$$
\begin{aligned}
& H_{2}=\operatorname{diag}(4(n+1), 4(n+1), \ldots, 4(n+1)), \\
& H_{3}=\operatorname{diag}(2(n+1), 2(n+1), \ldots, 2(n+1)) .
\end{aligned}
$$

Therefore, we find that $\mathcal{H}(\mathcal{P})$ has the following eigenvalues:

$$
\begin{aligned}
& \lambda_{11}=0, \quad \lambda_{22}=n+3, \quad \lambda_{33}=\cdots=\lambda_{n n}=2(n+1), \\
& \lambda_{i j}=4(n+1), \quad \lambda_{i n}=2(n+1), \quad \forall i, j \in\{1, \ldots, n-1\}, i \neq j .
\end{aligned}
$$

Therefore, $\mathcal{P}$ is parabolic and reaches a minimum $\mathcal{P}\left(h^{c}\right)=0$ for the solution $h^{c}$ of the system (3.5). It follows that $\mathcal{P} \geq 0$, and, hence,

$$
2 \tau \leq \frac{1}{2} n(n-1) \mathcal{C}+\frac{1}{2}(n+1)(n-1) \mathcal{C}(L)+n(n-1) c-2(n-1) \operatorname{trace}(\alpha) .
$$

Hence, we deduce that

$$
\rho \leq \frac{1}{2} \mathcal{C}+\frac{n+1}{2 n} \mathcal{C}(L)+c-\frac{2}{n} \operatorname{trace}(\alpha)
$$

for every tangent hyperplane $L$ of $M$. Taking the infimum over all tangent hyperplane $L$, the theorem trivially follows.

Moreover, we can easily check that the equality sign holds in the theorem if and only if

$$
h_{i j}^{\alpha}=0, \quad \forall i, j \in\{1, \ldots, n\}, i \neq j \text { and } \alpha \in\{n+1, \ldots, m\}
$$

and

$$
h_{n n}^{\alpha}=2 h_{11}^{\alpha}=\cdots=2 h_{n-1 n-1}^{\alpha}, \quad \forall \alpha \in\{n+1, \ldots, m\} .
$$

From (3.6) and (3.7), we conclude that the equality holds if and only if the submanifold $M$ is invariantly quasi-umbilical with trivial normal connection in $N$, such that with respect to 
suitable orthonormal tangent and normal orthonormal frames, the shape operators take the forms below:

$$
A_{n+1}=\left(\begin{array}{cccccc}
2 a & 0 & 0 & \cdots & 0 & 0 \\
0 & 2 a & 0 & \cdots & 0 & 0 \\
0 & 0 & 2 a & \cdots & 0 & 0 \\
\vdots & \vdots & \vdots & \ddots & \vdots & \vdots \\
0 & 0 & a & \cdots & 2 a & 0 \\
0 & 0 & a & \cdots & 0 & a
\end{array}\right), \quad A_{n+2}=\cdots=A_{m}=0
$$

Remark We have a slightly modified coefficient in the definition of $\delta_{C}(n-1)$; in fact, it was used the coefficient $\frac{n+1}{2 n(n-1)}$, as in $[22,23,25]$, instead of $\frac{n+1}{2 n}$, like in the present paper because we are working on the generalized normalized $\delta$-Casorati curvature $\delta_{C}(r ; n-1)$ for a positive real number $r \neq n(n-1)$, as in [24].

\section{Competing interests}

The authors declare that they have no competing interests.

\section{Authors' contributions}

All authors contributed equally and significantly in writing this paper. All authors read and approved the final manuscript.

\section{Author details}

${ }^{1}$ Department of Mathematics, Kyungpook National University, Daegu, 702-701, South Korea. ${ }^{2}$ Department of Mathematics Education and RINS, Gyeongsang National University, Jinju, 660-701, South Korea. ${ }^{3}$ Department of Mathematics Education, Busan National Univeristy of Education, Busan, 611-736, South Korea.

\section{Acknowledgements}

The authors would like to thank the referee for his valuable comments and suggestions which helped to improve the paper.

Received: 17 May 2014 Accepted: 30 July 2014 Published: 01 Sep 2014

\section{References}

1. Friedmann, A, Schouten, JA: Über die Geometrie der halbsymmetrischen Übertragungen. Math. Z. 21, $211-223$ (1924)

2. Hayden, HA: Subspaces of a space with torsion. Proc. Lond. Math. Soc. 34, 27-50 (1932)

3. Yano, K: On semi-symmetric metric connection. Rev. Roum. Math. Pures Appl. 15, 1579-1586 (1970)

4. Imai, T: Hypersurfaces of a Riemannian manifold with semi-symmetric metric connection. Tensor 23, 300-306 (1972)

5. Imai, T: Notes on semi-symmetric metric connections. Tensor 24, 293-296 (1972)

6. Nakao, Z: Submanifolds of a Riemannian manifold with semi-symmetric metric connections. Proc. Am. Math. Soc. 54, 261-266 (1976)

7. Chen, B-Y: Some pinching and classification theorems for minimal submanifolds. Arch. Math. 60, 568-578 (1993)

8. Chen, B-Y: Relations between Ricci curvature and shape operator for submanifolds with arbitrary codimensions Glasg. Math. J. 41, 33-41 (1999)

9. Chen, B-Y: A general inequality for submanifolds in complex space forms and its applications. Arch. Math. 67(6), 519-528 (1996)

10. Chen, B-Y: An optimal inequality for CR-warped products in complex space forms involving CR $\delta$-invariant. Int. J. Math. 23(3), 1250045 (2012)

11. Oiagă, A, Mihai, I: B.-Y. Chen inequalities for slant submanifolds in complex space forms. Demonstr. Math. 32, 835-846 (1999)

12. Mihai, A, Özgür, C: Chen inequalities for submanifolds of real space forms with semi-symmetric metric connection. Taiwan. J. Math. 14, 1465-1477 (2010)

13. Mihai, A, Özgür, C: Chen inequalities for submanifolds of complex space forms and Sasakian space forms endowed with semi-symmetric metric connections. Rocky Mt. J. Math. 41, 1653-1673 (2011)

14. Özur, C, Murathan, C: Chen inequalities for submanifolds of a locally conformal almost cosymplectic manifold with a semi-symmetric metric connection. An. Univ. 'Ovidius' Constanţa, Ser. Mat. 18(1), 239-253 (2010)

15. Özur, C, Murathan, C: Chen inequalities for submanifolds of a cosymplectic space form with a semi-symmetric metric connection. An. Univ. 'Ovidius' Constanţa, Ser. Mat. 58(2), 395-408 (2012)

16. Zhang, P, Zhang, L, Song, W: Chen's inequalities for submanifolds of a Riemannian manifold of quasi-constant curvature with a semi-symmetric metric connection. Taiwan. J. Math. (in press). doi:10.11650/tjm.18.2014.4045

17. Albertazzi, L: Handbook of Experimental Phenomenology: Visual Perception of Shape, Space and Appearance. Wiley, Chichester (2013) 
18. Casorati, F: Mesure de la courbure des surfaces suivant l'idée commune. Ses rapports avec les mesures de courbure gaussienne et moyenne. Acta Math. 14(1), 95-110 (1890)

19. Haesen, S, Kowalczyk, D, Verstraelen, L: On the extrinsic principal directions of Riemannian submanifolds. Note Mat. 29(2), 41-53 (2009)

20. Verstraelen, L: The geometry of eye and brain. Soochow J. Math. 30(3), 367-376 (2004)

21. Verstraelen, L: Geometry of submanifolds I. The first Casorati curvature indicatrices. Kragujev. J. Math. 37(1), 5-23 (2013)

22. Decu, S, Haesen, S, Verstraelen, L: Optimal inequalities involving Casorati curvatures. Bull. Transylv. Univ. Braşov, Ser. B 14(49), suppl., 85-93 (2007)

23. Decu, S, Haesen, S, Verstraelen, L: Optimal inequalities characterising quasi-umbilical submanifolds. J. Inequal. Pure Appl. Math. 9(3), Article ID 79 (2008)

24. Lee, JW, Vîlcu, GE: Inequalities for generalized normalized $\delta$-Casorati curvatures of slant submanifolds in quaternionic space forms. Preprint

25. Slesar, V, Şahin, B, Vîlcu, GE: Inequalities for the Casoratic curvatures of slant submanifolds in quaternionic space forms. J. Inequal. Appl. 2014, 123 (2014)

26. Blair, D: Quasi-umbilical, minimal submanifolds of Euclidean space. Simon Stevin 51, 3-22 (1977)

10.1186/1029-242X-2014-327

Cite this article as: Lee et al.: Optimal inequalities for the Casorati curvatures of submanifolds of real space forms endowed with semi-symmetric metric connections. Journal of Inequalities and Applications 2014, 2014:327

\section{Submit your manuscript to a SpringerOpen ${ }^{\circ}$ journal and benefit from:}

- Convenient online submission

- Rigorous peer review

- Immediate publication on acceptance

- Open access: articles freely available online

- High visibility within the field

- Retaining the copyright to your article 